https://artnodes.uoc.edu

\title{
La condición transreal: información expandida y hacktivismo en el Media Art $^{1}$
}

\author{
Carolina Fernández-Castrillo \\ Universidad Carlos III de Madrid (TECMERIN) \\ Università Ca' Foscari Venezia (Venice Centre for Digital and Public Humanities)
}

Fecha de presentación: septiembre de 2020

Fecha de aceptación: marzo de 2021

Fecha de publicación: julio de 2021

\section{Cita recomendada}

Fernández-Castrillo, Carolina. 2021. «La condición transreal: información expandida y hacktivismo en el Media Art». Artnodes, núm. 28. UOC. [Consulta: dd/mm/aa]. http://doi.org/10.7238/a. v0i28.377879

@ Cos textos publicados en esta revista están sujetos -si no se indica lo contrario- a una licencia de
SOMMERIGHISRESERVED
Reconocimiento 4.0 Internacional de CreativeCommons. La licencia completa se puede consultar en
https://creativecommons.org/licenses/by/4.0/deed.es_ES.

\section{Resumen}

Desde el Media Art hallamos una larga serie de expresiones culturales de carácter innovador en respuesta a las nuevas inquietudes e intereses surgidos en la era de la posverdad. En la presente investigación se hará especial hincapié en el importante papel del hacktivismo para incentivar la participación ciudadana en la lucha contra la desinformación y el abuso de poder institucional, económico o político-fáctico desde el ámbito más experimental de la cultura digital. Adoptaremos como objeto de estudio el corpus artístico de Paolo Cirio, por su interés al abordar el papel central

1. La presente investigación forma parte del proyecto «Digital Media Culture: Intercreativity and Public Engagement» del que la autora es investigadora principal en el Venice Centre for Digital and Public Humanities, Università Ca' Foscari Venezia (Ministero dell'Università e della Ricerca, Progetto MIUR Dipartimenti di Eccellenza). Los resultados del trabajo también se integran en la línea de investigación «Arqueología de medios digitales: intermedialidad, narrativas transmedia y CGU» que la investigadora lidera dentro del grupo TECMERIN (Televisión-cine: memoria, representación e industria, UC3M). 


\title{
artnodes
}

https://artnodes.uoc.edu

La condición transreal: información expandida y hacktivismo en el Media Art

de la información como medio y contenido creativo. En sus obras, la activación de originales mecanismos de visualización de datos e interacción con el usuario va dirigida no solo a denunciar injusticias y falsedades, sino también a estimular una intervención real sobre los propios hechos. A partir del análisis de algunas de sus principales contribuciones, se establecerá un marco teórico original para la identificación de aquellas aportaciones creativas que entran dentro de la que denominaremos «condición transreal». La relevancia de esta propuesta reside en el planteamiento de unas coordenadas para el estudio de aquellos proyectos transmedia de carácter interactivo, y en la mayoría de las ocasiones contestatario, capaces de brindar un nuevo modelo de relación con la audiencia y una atractiva estrategia de expansión del universo informativo desde el compromiso social mediante una acción directa sobre la realidad.

\section{Palabras clave}

crowdsourcing, hacktivismo, Media Art, narrativas transmedia, posverdad, visualización de datos

\section{The Transreal Condition: Expanded Information and Hacktivism in Media Art}

\begin{abstract}
Scores of innovative cultural expressions that address the new concerns and interests emerging in the "post-truth" era can be found in Media Art. In this research, special emphasis will be placed on the important role of hacktivism to encourage citizen participation in the fight against misinformation and the abuse of institutional, economic and political-factual power within the realm of experimentation in digital culture. The focus of this study will be Paolo Cirio's oeuvre, based on his interest in addressing the central role of information as a medium as well as its status as creative content. In his works, the activation of data visualisation mechanisms and the interaction with the user is aimed not only at denouncing injustices and falsehoods, but also at stimulating real intervention on the facts themselves. Starting with an analysis of some of Cirio's main productions, an original theoretical framework will be established to identify creative contributions that fall within what we may call the "transreal condition". The key of this proposal resides in proposing coordinates for the study of transmedia projects of an interactive and often rebellious nature, projects capable of not only ushering in a new model of audience engagement but also expanding the information field beyond the social contract through direct action into reality.
\end{abstract}

\section{Keywords}

crowdsourcing, data visualization, hacktivism, media art, post-truth, trans-media storytelling

\section{Introducción}

El aprendizaje obtenido durante las dos primeras décadas del siglo XXI a través del uso de las redes sociales ha derivado en el establecimiento de una nueva relación con la propia realidad, expandida a través de la transmedialidad o del, más reciente, universo metainformativo del blockchain. Este horizonte abre nuevas vías para la visualización de datos e, incluso, la intervención directa sobre la realidad, activando así los mecanismos para la puesta en marcha de una democracia radical (Laclau y Mouffe, 2001). Se trata de una forma de acción presente en la obra del artista hacker Paolo Cirio que saltó a la primera línea del Media Art con Loophole for All (2013), obra galardonada en el año 2014 con el Golden Nica en la categoría de arte interactivo del Festival Ars Electronica, el mayor reconocimiento internacional en el mundo de la cultura digital. Se trataba de una controvertida propuesta con la que el italiano pretendía dar visibilidad a los resultados de su investigación sobre evasión fiscal en las Islas Caimán. Mediante una insólita estrategia de visualización de la información obtenida, por un lado, revelaba la identidad de los delincuentes y, por otro, proporcionaba al usuario las claves para aprender a evadir impuestos siguiendo el mismo modus operandi.

A través de una revisión de las aportaciones de Cirio, introduciremos el concepto de "condición transreal», un área interdisciplinar donde hacktivismo, narrativas transmedia e innovación digital se unen en la generación de originales experiencias participativas para el acceso a la información y, aún más importante, la acción directa sobre la realidad. Siguiendo la estela de otras corrientes anteriores como el arte investigador (Investigatory Art) o el más genérico arte informativo (Info Arts), una vez determinadas las características fundamentales de los proyectos englobados bajo esta nueva categoría creativa, se procederá a su definición para facilitar así su identificación. 


\section{artnodes}

https://artnodes.uoc.edu

\section{Una aproximación al arte investigador e informativo}

El arte investigador surge a finales de la década de los años sesenta como instrumento de concienciación y protesta social para aportar, desde un enfoque crítico, una mayor visibilidad y conocimiento acerca de los mecanismos que rigen el poder institucional (Shanken, 2012). Se trata de un sector creativo muy próximo al de la investigación periodística, pues el artista parte de una exhaustiva indagación sobre un determinado tema para desvelar información inédita. A diferencia del reportero, el creador difunde los resultados mediante la producción de una obra artística de alto componente estético-conceptual poniendo el foco de atención sobre determinadas realidades ocultas a la mayoría de la población y cuyo fin último es la activación de una serie de reacciones por parte de la sociedad, promoviendo así el cambio.

En este tipo de propuestas artísticas, el intercambio de información se basa en la inmediatez y la interactividad. Por tanto, tal y como señala Edward E. Shanken² (2012), las experiencias generadas en tiempo real adquieren una mayor relevancia. Por ello, el componente tecnológico ha ido ganando protagonismo en el arte investigador a lo largo de las últimas décadas. Artistas como Hans Haacke fueron de los primeros en invitar al público a completar sus obras mediante la aportación de una serie de datos personales para conocer el perfil de la audiencia sin mediación institucional alguna. En el caso de su célebre Visitor's Profile (1970) se trataba de rellenar un cuestionario sobre eventos culturales a través de un ordenador y los resultados estadísticos se proyectaban en tiempo real en la sala de exposiciones. Según el propio Haacke, «los visitantes producían un retrato colectivo mediante un proceso participativo y autorreflexivo» ${ }^{3}$ (2009). Este es uno de los primeros ejemplos de recolección, procesamiento y visualización de datos en tiempo real y, al mismo tiempo, también constituye una intervención crítica y directa en el propio espacio objeto de reflexión.

Al contrario que el arte informativo (Wilson, 2002), en el arte investigador la exposición de contenidos inéditos y, en la mayoría de las ocasiones de carácter sensible, supone un requisito fundamental. A la primera categoría pertenecen obras centradas en el propio medio como vehículo de transmisión de información, que no necesariamente brindan datos desconocidos sobre un tema concreto de interés social. Tomemos como ejemplo la adopción del código de barras como icono de la reducción de los individuos a meros bloques de información en las obras de Perry Hoberman (Bar Code Hotel, 1994) y Paul Vanouse (Items 1-2,000, 1997). 0, también en la década de los noventa,
La condición transreal: información expandida y hacktivismo en el Media Art

las aportaciones de artistas como Bruce Cannon (Ten Things I Can Count On, 1997-1999), Simon Penny (Fugitive 2, 1996-1997), Joel Slayton (Telepresent Surveillance, 1995) o Julia Scher (Predictive Engineering, 1993), al ámbito de la vigilancia. En ambos casos, la alienante experiencia de pasar a formar parte de la esfera de datos y la correspondiente pérdida de identidad adquieren mayor relevancia que el contenido en sí.

El principal punto de encuentro entre el arte investigador y el arte informativo es el interés común por la visualización de datos basada, en la mayoría de las ocasiones, en la puesta en marcha de procesos interactivos. El creciente establecimiento de alianzas entre el sector científico y el artístico constituye un terreno fértil para este tipo de experimentaciones, donde encontramos la contribución de Felice Frankel como artista residente en el MIT o Rachel Strickland, experta en la creación de instalaciones interactivas de corte antropológico. También resulta destacable la labor de Donna Cox, colaboradora del Centro Nacional Supercomputacional de la Universidad de Illinois y premiada por la prestigiosa revista Leonardo, por «hacer visibles las complejas, y aún invisibles, estructuras del universo» (Leonardo, 2014). Asimismo, desde el grupo de estética computacional (también del MIT), John Maeda ha puesto en marcha su investigación sobre el desarrollo de sistemas de relaciones visuales para expresar de forma intuitiva los distintos grados de relevancia de la información de tipo cualitativo.

En el arte investigador, dar visibilidad a los contenidos de forma atractiva es un aspecto importante, pero lo es más aún el conseguir destapar escándalos 0 verdades incómodas relacionadas con desigualdades, la vulneración de los derechos humanos, la corrupción en las relaciones institucionales o el abuso de poder por parte de las autoridades. Paolo Cirio muestra interés por estos temas, que, además, actualiza a través de sus investigaciones sobre el uso indebido de las nuevas tecnologías por parte de los agentes sociales más poderosos. Uno de los factores más singulares de la obra del artista italiano consiste en la concepción de amplios proyectos de carácter intermedial e interactivo en los que sus conocimientos como hacker le proporcionan acceso a información anteriormente «invisible», al tiempo que consigue promover la sublevación por parte de los usuarios a través de una intervención directa en la realidad. El hacktivismo es, por tanto, un aspecto decisivo para comprender el revolucionario planteamiento de Cirio y también la clave fundamental para definir la condición transreal como resultado natural de la evolución del arte investigador en la era postdigital.

2. A partir de las aportaciones de Jack Burnham (1969) o Caroline Jones (2012) sobre la incorporación de los «sistemas en tiempo real» al ámbito artístico, Edward E. Shanken sostiene que desde mediados de los años noventa, el arte contemporáneo brinda nuevas posibilidades a los usuarios para el acceso y la manipulación de datos anteriormente inaccesibles sobre las complejas (y a menudo ocultas) relaciones sociales. Una enriquecedora aportación desde el ámbito de la creación artística que, según Shanken, se basa en mecanismos similares a los de la cultura de red (2012).

3. Todas las traducciones al castellano en las citas de este estudio han sido realizadas por la autora del presente artículo. 


\section{artnodes}

https://artnodes.uoc.edu

\section{Hacktivismo y Media Art}

Furtherfield (2012) sostiene que la palabra «hacktivismo» fue empleada por primera vez en 1995 por el escritor Jason Sack ${ }^{4}$ para definir una pieza de Media Art de Shu Lea Cheang en un artículo publicado en InfoNation. En las últimas décadas han sido numerosos los intentos por definir este término desde el ámbito académico. Dorothy Denning (2001) concibe el hacktivismo de forma genérica como el resultado de la alianza entre activismo y piratería, eso sí, sin ocasionar daños graves. Mark Milone recurre a esta palabra para referirse al activismo on-line basado en el «acceso subrepticio a ordenadores 0 la diseminación de software potencialmente disruptivo o subversivo» (2002: 77). A pesar de servirse de recursos similares a los del hacker, Milone señala que el hacktivista pretende llamar la atención sobre determinados objetivos políticos o sociales, en ocasiones llegando a incurrir en la criminalidad. Otros autores también centran sus aportaciones en la naturaleza política de este fenómeno, como Tim Jordan y Paul A. Taylor (2004). Sin embargo, este tipo de aproximaciones no subrayan la distancia entre hacktivismo y ciberterrorismo, o las manifestaciones activistas on-line de carácter no disruptivo. En su tesis doctoral, Alexandra Samuelson (2004) identifica precisamente la no violencia y la transgresión on-line como elementos distintivos del hacktivismo.

Este fenómeno resulta de interés para un amplio número de disciplinas: ciencias de la información, económicas y políticas, sociología, derecho, filosofía, ciberseguridad, estudios culturales, etc. Por lo general, las investigaciones sobre hacktivismo se han centrado en dos áreas principales: la ciberseguridad y la desobediencia civil. En este último ámbito, el papel de los medios de comunicación adquiere un especial protagonismo. De hecho, según Denning, en buena parte se persigue que el activista y sus causas adquieran publicidad (2001: 263).

En cuanto a los perfiles de los hacktivistas, van desde la figura del hacker programador a la del artista-activista. Por lo general, el primero ve al segundo como un ignorante, desconocedor de la infraestructura de internet y técnicamente incompetente; mientras que el segundo ve al primero como parte de la élite tecnológica, insensible a las necesidades sociales (Samuelson, 2004: 39). En este estudio nos centraremos en el artista-activista como agente de cambio social en defensa de los derechos fundamentales y en su aportación tanto al arte digital experimental como al ámbito mediático. Ciertamente, en este tipo de obras se pone en valor el uso estético y ético de la información como el principal material y medio artístico. También recordemos que el arte de los nuevos medios hace referencia a todas aquellas aportaciones artísticas creadas a partir de las nuevas tecnologías y los medios de comunicación emergentes.
La condición transreal: información expandida y hacktivismo en el Media Art

En su ensayo «Aesthetics Information Ethics», Cirio (2017) resumía los desafíos éticos con los que se había encontrado como artista hacker en su relación con la audiencia y con los agentes sociales protagonistas de sus obras. En su labor como artista y activista, el italiano ha abordado temas tan candentes como el derecho a la privacidad on-line, la censura, la vigilancia, las fake news, la piratería, la cultura hacker o el poder inherente al uso de algoritmos, big data o filtraciones en la red. La visualización de los datos y la interacción tanto con el usuario como con los agentes sociales, instituciones y poderes públicos presentes en sus obras nos obliga a reflexionar sobre la complejidad ética de este tipo de relaciones.

\section{Hacia una definición de la "condición transreal»}

En la década de los años sesenta emerge el arte intervencionista, una corriente próxima al arte conceptual y de performance de la que extraeremos una serie de ideas para abordar los orígenes de la condición transreal. En 1960 Guy Debord puso de relieve la pasividad del espectador y desde el Artist Placement Group (APG) se sugería que el artista adoptara un papel más activo dentro del contexto político y social. Debía alejarse de las galerías para adentrarse en el sector industrial y gubernamental y efectuar así una serie de cambios. Por lo tanto, «El término "intervención artística" se aplica al arte diseñado específicamente para interactuar con una estructura o situación existente, ya sea otra obra de arte, la audiencia, una institución o en el dominio público» (Tate, s.d.).

A diferencia del arte intervencionista, la condición transreal surge en buena parte como resultado del aprendizaje adquirido a través del hacktivismo, por lo que también acoge aquellas intervenciones sobre el código digital de la propia realidad objeto de interés. Este nuevo concepto que introducimos en esta investigación es el fruto de la evolución de la visualización de la información, la interacción con los datos y el papel del artista-activista en la era postdigital. La elección del prefijo -trans responde a la idea de trascender la mera representación de los acontecimientos, interviniendo directamente desde su matriz, traspasando así los límites entre la dimensión analógica y digital de la propia realidad objeto de estudio.

En la condición transreal se contempla la publicación de información inédita a partir de una investigación original, pero, al contrario que en el arte investigador, hay una acción directa sobre la realidad a través de dinámicas características de la cultura hacker y, al mismo tiempo, llevando a cabo una serie de acciones off-line. Bajo esta

4. En su texto, Furtherfield (2012) cita a Jason Sack, mientras que al revisar el ejemplar original del artículo publicado en InfoxNation figura como autor Jason Logan (1995). Tal y como aclara en su blog Jericho (2014), se trata de un pseudónimo. 


\section{artnodes}

https://artnodes.uoc.edu

denominación se engloban las creaciones que desde finales de la segunda década del siglo xxı han ido emergiendo bajo el influjo de las corrientes intercreativas ${ }^{5}$ presentes en las redes sociales. De hecho, uno de los elementos esenciales de la condición transreal es la incorporación de acciones colectivas mediante la activación de procesos colaborativos basados en la creación de contenidos generados por los usuarios. Por último, dentro de las dinámicas participativas, cabe destacar también la promoción de una fuerte concienciación social y la presión a los grupos de poder con la intención de ejecutar un cambio real.

En el análisis de aquellos proyectos susceptibles de formar parte de la condición transreal, debe tenerse en cuenta si se lleva a cabo una expansión del universo informativo a través de las siguientes fases:

1. Búsqueda de información inédita.

2. Visualización de los contenidos.

3. Interacción con el produsuario.

4. Acción directa sobre la realidad.

El hacktivismo está presente de forma transversal en cada una de las etapas de la obtención, elaboración y difusión de la realidad expandida. Una vez identificado el fenómeno que centrará el acto de denuncia social, arranca el proceso de investigación a partir de la esfera de lo invisible (pensemos en los resultados obtenidos a través de la minería de datos, fact-checking, inteligencia de fuentes abiertas, etc.) para la recopilación, traducción, reproducción y posterior difusión de los datos obtenidos. En cuanto a su visualización, existen múltiples opciones, desde aquellas más próximas al software $a^{6}{ }^{6}$, entendido como el «arte de lo imperceptible» (Parikka, 2010), previamente desarrollado en las obras de grupos como Jodi. org 0 0100101110101101.org, a la producción de documentales y reportajes, sitios web, performances e intervenciones en plataformas digitales y espacios públicos, etc. En definitiva, se trata de generar una estrategia transmedia de carácter provocador que permita al produsuario ${ }^{7}$ interactuar desde alguno de los puntales de creación y difusión de contenidos para contribuir a esa extensión informativa. Por último, se contempla una intervención directa sobre la realidad objeto de interés. Precisamente aquí reside la característica distintiva de la condición transreal: la puesta en marcha de una serie de acciones de oposición para combatir cualquier tipo de abuso tanto en su dimensión analógica como digital, siempre en favor de la sociedad en su conjunto, nunca para el beneficio propio o tan solo de unos pocos.
La condición transreal: información expandida y hacktivismo en el Media Art

A diferencia de otras experiencias artísticas de hacktivismo, net. art 0 software art, en la condición transreal la esfera informativa es una constante ineludible, al igual que el planteamiento de una clara estrategia intervencionista estructurada según las cuatro fases anteriormente citadas. Lo que sí tienen en común con los hackers y grupos radicales es el haber descubierto que el grado de vulnerabilidad de las instituciones es mayor en el ciberespacio que en el mundo físico (Harmon, 1998). Algo de lo que también es consciente Paolo Cirio, el principal exponente de la condición transreal en la actualidad.

\subsection{Más allá de la interacción y visualización de la información} Desde la comunicación social, en las últimas décadas ha surgido la necesidad de transmitir la información mediante estrategias que consientan una mayor fluidez narrativa y visual (McKenna et al., 2017), favoreciendo así nuevos tipos de interacción con el usuario. La condición transreal plantea, además, un avance significativo en su relación con la propia información, al convertirse en agente activo del proceso de obtención y difusión de los datos. Asimismo, se abre una nueva línea de acción en la que la alianza entre medios de comunicación y hacktivismo puede contribuir al avance social mediante la denuncia de fraudes e injusticias. Tomemos como ejemplo el caso de Capture (2020), una obra en la que Cirio denuncia el uso de la tecnología que consiente el reconocimiento facial sin previa autorización. A continuación, comprobaremos el cumplimiento de las cuatro fases de la condición transreal.

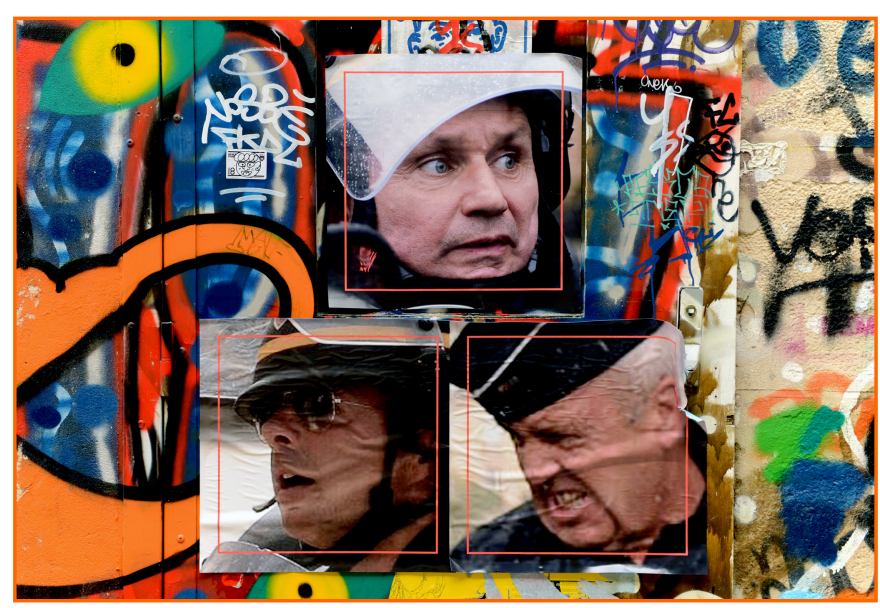

Figura 1. Acción de arte urbano del proyecto Capture. París (Francia), 2020. Fotografía captada por Florian Draussin y cedida por Paolo Cirio

5. Tim Berners-Lee (1997 y 1999) fue quien acuñó el término «intercreatividad».

6. Para una mayor profundización sobre el software art, se sugiere revisar las aportaciones de Andreas Broeckmann (2003), Rachel Greene (2004), Inke Arns (2005), Matthew Fuller (2006) y Geoff Cox (2007), entre otros.

7. El neologismo "produsuario» fue introducido por Axel Bruns (2008) en su libro Blogs, Wikipedia, Second Life, and Beyond: From Production to Produsage para referirse a la evolución natural en la era de la web 2.0 del concepto de «prosumidor» (Toffler, 1980), esa figura intermedia entre productor y consumidor. El término "produsuario» refleja el papel del usuario como productor de información y agente activo en las dinámicas participativas que tienen lugar en las interacciones comunicativas a través de internet. 


\section{artnodes}

https://artnodes.uoc.edu

1. Búsqueda de información inédita: el artista recopiló un millar de imágenes de policías tomadas en espacios públicos durante una serie de manifestaciones en Francia, difundidas a través de los medios de comunicación o simplemente disponibles en internet. Posteriormente, las procesó con un software de reconocimiento facial.

2. Visualización de los contenidos: Cirio imprimió las fotos de los oficiales y realizó una serie de intervenciones de arte urbano, exponiendo dicha información por las calles de París. Desde su web lanzó varios ensayos sobre la obra (Cirio, 2020a y c) y la campaña contra el reconocimiento facial (Cirio, 2020b). A la estrategia transmedia también se sumaron materiales audiovisuales de corte documental, entrevistas, manifiestos y la creación de una instalación fotográfica programada para la exposición Panorama 22 en Le Fresnoy (Tourcoing, Francia), que finalmente fue censurada por el Gobierno francés en octubre de 2020. Una noticia que en las semanas posteriores siguió ocupando los titulares de numerosas cabeceras internacionales ${ }^{8}$ y que contribuyó así a la difusión del proyecto y a dar una mayor visibilidad a la propia campaña.

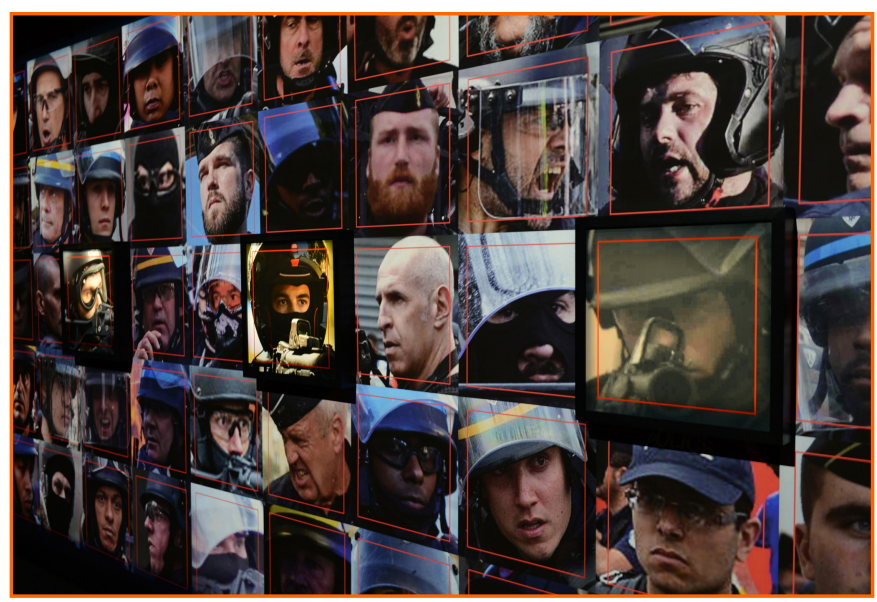

Figura 2. Instalación Capture. Le Fresnoy, Tourcoing (Francia), 2020. Fotografía cedida por Paolo Cirio

3. Interacción con el produsuario: a través de una plataforma on-line titulada Capture-Police.com, Cirio creó una base de datos de los 4.000 rostros de policías franceses para activar un proceso de crowdsourcing dirigido a la identificación de sus nombres por parte de los usuarios. Los resultados se hicieron públicos una vez llevadas a cabo las verificaciones oportunas.
La condición transreal: información expandida y hacktivismo en el Media Art

4. Acción directa sobre la realidad:por último, el italiano ha puesto en marcha la campaña \#Ban Facial Recognition Europe (2020) contra el potencial abuso del reconocimiento facial y de la inteligencia artificial en Europa con la colaboración de organizaciones en defensa de los derechos fundamentales en la era digital, como por ejemplo EDRi (European Digital Rights).

En Capture se denuncian las asimetrías de poder, invirtiendo así de forma provocadora el paradigma del poderoso que observa al indefenso para generar un debate social que posibilite una mejora en la regulación del uso de las nuevas tecnologías. El enfoque ético, político y estético de este proyecto, según señala Cirio, «apunta a resolver la perplejidad sobre el sentido de transparencia, privacidad y autonomía de ciudadanos y autoridades» (2020c). Se señala, por tanto, la falta de una política concreta en materia de privacidad en el uso de dichas tecnologías por parte de las autoridades.

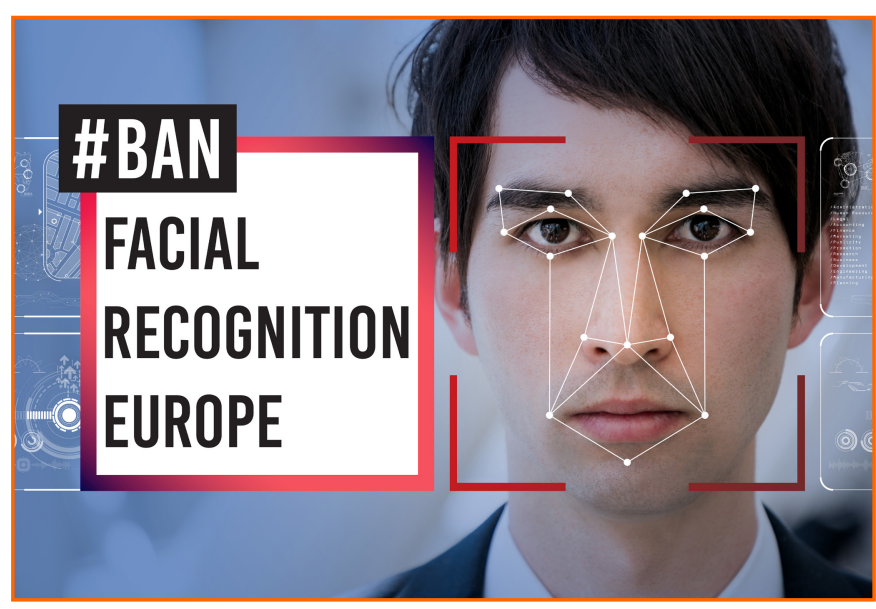

Figura 3. Banner publicitario de la campaña \#Ban Facial Recognition Europe. Fotografía cedida por Paolo Cirio

En una reciente entrevista ${ }^{9}$, el propio artista reflexionaba acerca de su posicionamiento como hacktivista dentro del ámbito del Media Art. Cirio sostiene la existencia de una tensión generacional aún irresuelta entre el tecnoanarquismo de los años noventa y comienzos del siglo xxı frente a la búsqueda de un nuevo sistema de regulación para alcanzar un cambio social. Tal y como él mismo explica, más allá de un mero ataque antisistema: el hacktivismo «(...) se ha convertido en un método mediante el que consigo afrontar una cuestión compleja revelando datos sensibles, usándolos como provocación y esperando

8. Véanse, entre otros, «Activists Turn Facial Recognition Tools Against the Police», en The New York Times (Hill, 2020); «Au Fresnoy, 'Capture' de Paolo Cirio battu à plate censure», en Liberation (Mercier, 2020); "Des photos de policiers sur la place publique», en Le Figaro (Gonzalès, 2020); "Darmanin contraint un artiste italien à retirer des photos de policiers de son site», en Huffington Post (AFP, 2020), etc.

9. La autora del artículo entrevistó a Paolo Cirio en agosto de 2020. El artista adelantaba en exclusiva los objetivos fundamentales de Capture, una obra presentada a posteriori, en el mes de septiembre de ese mismo año. 


\section{artnodes}

https://artnodes.uoc.edu

una reacción por parte del sujeto de interés en la obra. Por último, en la fase final de intervención persigo un cambio de regulación. No se trata, por tanto, únicamente de generar una provocación en sí misma, sino de darle la vuelta a la ley para conseguir un uso positivo» ${ }^{10}$. Cirio reconoce una serie de puntos de encuentro entre este tipo de intervenciones y las dinámicas presentes en el desempeño de la labor periodística. El punto de partida es el mismo: la investigación en profundidad durante varios meses de la realidad objeto de estudio para la extracción de datos sensibles que puedan resultar de interés público. La publicación de esta información mediante un lenguaje claro es otro aspecto en común, al igual que la denuncia de realidades controvertidas para suscitar la atención de los ciudadanos y promover un cambio que afiance los derechos fundamentales. En la provocación reside el punto de mayor divergencia, pues el artista dispone de un amplio margen de acción para dar visibilidad a la información y promover nuevas formas de interacción con los usuarios, en ocasiones recurriendo incluso a prácticas ilegales.

Según comentaba en la conversación mantenida con la autora de este artículo, en 2018 Cirio sintió la necesidad de introducir el concepto de arte regulatorio (Regulatory Art) para englobar aquellas obras artísticas de carácter creativo, crítico y dinámico dirigidas a impulsar una gobernanza democrática mediante «el compromiso público, el control, la supervisión, la acción política, la propuesta de instrumentos jurídicos y el activismo legislativo» (Cirio, 20182020). Un avance más en su aportación como artista hacktivista a la relación entre información y Media Art, donde el debate sobre el marco legislativo abre un nuevo horizonte a tener en cuenta en futuras investigaciones.

\section{Conclusiones}

A lo largo del siglo xx, la experimentación con los medios de comunicación de masas permitió a las distintas vanguardias artísticas revelar aspectos desconocidos tanto del potencial creativo de las nuevas tecnologías como de su posible impacto en las dinámicas socioeconómicas y políticas. La puesta en marcha de prácticas de tipo radical en la crítica al sistema también ha sido un terreno fértil en términos de innovación comunicativa, proporcionando innovadoras fórmulas de expresión de gran utilidad incluso para los profesionales de la información (pensemos, por ejemplo, en la influencia del videoarte en el ámbito documental o el reporterismo). En la actualidad, las acciones artísticas de guerrilla llevadas a cabo por los hacktivistas contribuyen a tomar conciencia de la interrelación entre la esfera analógica y digital.

En la época postdigital el universo del big data sigue siendo aún desconocido e inaccesible para la mayoría de la ciudadanía;
La condición transreal: información expandida y hacktivismo en el Media Art

sin embargo, determina en gran medida múltiples aspectos de la realidad cotidiana. En esta nueva fase se trasciende la interfaz, en lo relativo a la permanente actualización de nuevos mecanismos para la visualización de la información y la interacción con el usuario, para adentrarnos en el propio código digital como fuente de obtención de datos y, al mismo tiempo, espacio de intervención para el cambio social. Este giro epistemológico obliga a una revisión de los procesos de acceso a la información, su consumo y las dinámicas de participación en el ecosistema digital. Por tanto, acciones como las llevadas a cabo por Paolo Cirio desde el Media Art suponen un punto de referencia para comprender esta importante transición para hallar nuevas fórmulas que garanticen una mayor transparencia y democratización en el acceso a la información.

Casos como la internet oscura, Cambridge Analytica, el espionaje a través de redes sociales, la policía predictiva o el derecho al olvido requieren de una reflexión por parte de todos los protagonistas sociales y no únicamente de las grandes compañías o gobiernos de turno. A través de sus aportaciones, Cirio demuestra que el arte puede y debe incitar a considerar delicadas cuestiones éticas relativas al papel de la información en la actualidad, Ilegando a estimular la participación ciudadana y la propia intervención en la realidad para ejercer un auténtico cambio social.

\section{Referencias bibliográficas}

Agence France-Presse. 2020. «Darmanin contraint un artiste italien à retirer des photos de policiers de son site». The Huffington Post, 3 octubre, 2020. [Fecha de consulta: 10/12/2020] https:// www.huffingtonpost.fr/entry/darmanin-contraint-un-artisteitalien-a-retirer-des-photos-de-policiers-de-son-site_fr_5f7 7bab0c5b64b480aade1f6?ncid=tweetInkfrhpmg00000001

Arns, Inke. 2005. "Code as Performative Speech Act». Artnodes, 4: 1-9. [Fecha de consulta: 10/12/2020] https://www.raco. cat/index.php/Artnodes/article/view/53082

Ban Facial Recognition Europe. 2020. \#Ban Facial Recognition Europe. Página web oficial de la campaña. [Fecha de consulta: 10/12/2020] https://ban-facial-recognition.wesign.it/ droitshumains/ban-facial-recognition-europe

Berners-Lee, Tim. 1997. «Realising the full potential of the web». Página web oficial de World Wide Web Consortium. [Fecha de consulta: 10/12/2020] http://www.w3.org/1998/02/Potential. html

Berners-Lee, Tim. 1999. Weaving the Web. Nueva York: HarperCollins. 


\section{artnodes}

https://artnodes.uoc.edu

Broeckmann, Andreas. 2003. «0n Software as Art». Sarai Reader. Media, Information, The Contemporary: 215-218. [Fecha de consulta: 10/12/2020] http://archive.sarai.net/files/original/ 07c3e0e280b4363d625093a1f5bce554.pdf

Bruns, Axel. 2008. Blogs, Wikipedia, Second Life, and beyond: from production to produsage. Nueva York: Peter Lang.

Burnham, Jack. 1969. «Real Time Systems». Artforum, 8(1): 49-55.

Cirio, Paolo. 2020a. Capture. Página web oficial de Paolo Cirio. [Fecha de consulta: 10/12/2020] https://www.paolocirio.net/ work/capture/

Cirio, Paolo. 2020b. «Text campaign and research Ban Facial Recognition Europe». Página web oficial de Paolo Cirio. [Fecha de consulta: 10/12/2020] https://www.paolocirio.net/press/ texts/text_capture_ban-facial-recognition.php

Cirio, Paolo. 2020c. «Text Project and artist's statements project Capture». Página web oficial de Paolo Cirio. [Fecha de consulta: 10/12/2020] https://www.paolocirio.net/press/texts/ text_capture.php

Cirio, Paolo. 2018-2020. «Regulatory Art». Página web oficial de Paolo Cirio. [Fecha de consulta: 10/12/2020] https://paolocirio. net/press/texts/text_regulatory-art.php

Cirio, Paolo. 2017. «Aesthetics of Information Ethics». Página web oficial de Paolo Cirio. [Fecha de consulta: 10/12/2020] https://paolocirio.net/press/texts/aesthetics-informationethics.php

Cox, Geoff. 2007. «Generator: The Value of Software Art». Issues in Curating Contemporary Art and Performance, editado por Judith Rugg, y Michèle Sedgwick, 147-162. Bristol: Intellect. [Fecha de consulta: 10/12/2020] https://monoskop.org/images/5/53/ Cox_Geoff_2007_Generator_The_Value_of_Software_Art.pdf

Denning, Dorothy E. 2001. «Activism, Hacktivism, and Cyberterrorism: the Internet As a Tool for Influencing Foreign Policy». Networks and Netwars: The Future of Terror, Crime, and Militancy, editado por John Arquilla, y David Ronfeldt, 239-287. Santa Monica, California: Rand. [Fecha de consulta: 10/12/2020] https://www.rand.org/pubs/monograph_reports/MR1382.html

Fuller, Matthew. 2006. Softness: Interrogability; General Intellect; Art Methodologies in Software. Huddersfield: Digital Research Unit at Huddersfield University.

Furtherfield. 2012. «Revisiting the Curious World of Art and Hacktivism». Página web oficial de Furtherfield. [Fecha de consulta: 10/12/2020] https://www.furtherfield.org/revisitingthe-curious-world-of-art-hacktivism/

Gonzalès, Paule. 2020. «Des photos de policiers sur la place publique». Le Figaro, 2 octubre, 2020. [Fecha de consulta: 10/12/2020] https://www.lefigaro.fr/actualite-france/desphotos-de-policiers-sur-la-place-publique-20201002

Greene, Rachel. 2004. Internet Art. Londres: Thames \& Hudson. Haacke, Hans. 2009. «Lessons Learned». Tate Papers, 12. [Fecha
La condición transreal: información expandida y hacktivismo en el Media Art

de consulta: 10/12/2020] https://www.tate.org.uk/research/ publications/tate-papers/12/lessons-learned

Harmon, Amy. 1998. "'Hacktivist' of All Persuasions Take Their Struggle to the Web». The New York Times, 31 octubre, 1998. [Fecha de consulta: 10/12/2020] https://www.nytimes. com/1998/10/31/world/hacktivists-of-all-persuasions-taketheir-struggle-to-the-web.html?pagewanted=all

Hill, Kashmir. 2020. «Activists Turn Facial Recognition Tools Against the Police». The New York Times, 27 octubre, 2020. [Fecha de consulta: 10/12/2020] https://www.nytimes.com/2020/10/21/ technology/facial-recognition-police.html

Jericho. 2014. "On the origins of the term 'Hacktivism'». Curmudgeonly Ways. Rants of a deranged squirrel, 17 febrero, 2014. [Fecha de consulta: 10/12/2020] https://jerichoattrition. wordpress.com/tag/jason-sack/

Jones, Caroline. 2012. «Systems Symptoms: Jack Burnham's 'Systems Esthetics'». Artforum, 51(1): 113-116.

Jordan, Tim, y Paul A. Taylor. 2004. Hacktivism and Cyberwars. Rebels with a cause. Londres y Nueva York: Routledge. DOI: https://doi.org/10.4324/9780203490037

Laclau, Ernesto; Chantal Mouffe. 2001. Hegemony and Socialist Strategy: Towards a Radical Democratic Politics. Londres: Verso. Leonardo. 2014. "The Leonardo Awards Program: Award Recipients». Página web oficial de Leonardo on-line. [Fecha de consulta: 10/12/2020] https://www.leonardo.info/isast/ awardwinners.html

Logan, Jason. 1995. «The Virtual Underground: Take the Skinheads Bowling». InfoNation, noviembre 1995: 9-10.

McKenna, Sean; Nathalie Henry Riche, Bongshin Lee, Jeremy Boy, y Miriah Meyer. 2017. «Visual narrative flow: Exploring factors shaping data visualization story reading experiences». Eurographics conference on visualization (EuroVis), 36(3): 377-387. [Fecha de consulta: 10/12/2020] http://sci.utah.edu/ vdl/ papers/2017_eurovis_narrative-flow.pdf DOl: https://doi. org/10.1111/cgf.13195

Mercier, Clémentine. 2020. «Au Fresnoy, 'Capture' de Paolo Cirio battu à plate censure». Libération, 12 octubre, 2020. [Fecha de consulta: 10/12/2020] https://next.liberation.fr/ arts/2020/10/12/au-fresnoy-capture-de-paolo-cirio-battua-plate-censure_1802147

Milone, Mark G. 2002. «Hacktivism: Securing the National Infrastructure». The Business Lawyer, 58(1): 383-413. [Fecha de consulta: 10/12/2020] https://www.arifyildirim.com/ilt510/mark.milone.pdf

Parikka, Jussi. 2010. «Ethologies of Software art:What can a digital body of code do?». Deleuze and Contemporary Art, editado por Zepke, Stephen, y Simon 0'Sullivan: 116-132. Edimburgo: Edinburgh University Press DOl: https://doi.org/10.3366/edinburgh/9780748638376.003.0008

Samuelson, Alexandra. 2004. Hacktivism and the Future of Political Participation. Tesis doctoral. Harvard University. [Fecha de con- 


\section{artnodes}

https://artnodes.uoc.edu

sulta: 10/12/2020] http://alexandrasamuel.com/dissertation/ pdfs/index.html

Shanken, Edward A. 2012. «Investigatory Art: Real-time systems and network culture». Necsus, 'Tangibility', noviembre, 2012. [Fecha de consulta: 10/12/2020] https://necsus-ejms.org/ investigatory-art-real-time-systems-and-network-culture/ DOI: https://doi.org/10.5117/NECSUS2012.2.SHAN
La condición transreal: información expandida y hacktivismo en el Media Art

Tate. s.d. «Art Intervention». [Fecha de consulta: 10/12/2020] https://www.tate.org.uk/art/art-terms/a/art-intervention

Toffler, Alvin. 1980. The third wave. Nueva York: Morrow.

Wilson, Stephen. 2002. Information Arts. Intersections of Arts, Science, and Technology. Cambridge, Mass.: MIT Press. D0I: https://doi.org/10.7551/mitpress/3765.001.0001 


\section{artnodes}

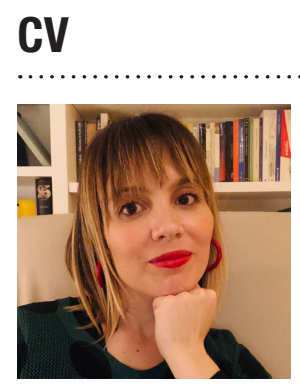

\section{Carolina Fernández-Castrillo}

Universidad Carlos III de Madrid (TECMERIN)

Università Ca' Foscari Venezia (Venice Centre for Digital

and Public Humanities)

Profesora Ayudante Doctora del Departamento de Comunicación (UC3M) en el área de Innovación Digital y Cibercultura. Actualmente es Visiting Scholar en Venice Centre for Digital and Public Humanities (Università Ca' Foscari Venezia), donde es la IP del proyecto de investigación «Digital Media Culture: Intercreativity and Public Engagement» (programa de excelencia MIUR, Ministero dell'Università e Ricerca). Su tesis doctoral sobre la Arqueología de Medios obtuvo Premio Extraordinario de Doctorado con Mención Europea (UCM y Sapienza Università di Roma) y la Mención de Excelencia del Real Colegio Complutense en Harvard. También ha sido Visiting Scholar en diversas instituciones internacionales: Yale University; Bayreuth Universität; Friedrich-Alexander-Universität Erlangen-Nürnberg; Internet Interdisciplinary Institute (IN3, UOC); o ZKM Center for Art and Media Karlsruhe, donde es Associate Researcher desde 2009.

www.carolinafcastrillo.com

ORCID ID: https://orcid.org/0000-0001-6108-6440 